\title{
ИСПОЛЬЗОВАНИЕ ДЕНОСУМАБА У БОЛЬНЫХ ПЕРВИЧНЫМ ГИПЕРПАРАТИРЕОЗОМ
}

\author{
Крюкова И.В., Древаль А.В., Тевосян Л.Х., Полякова Е.Ю., Барсуков И.А., Золотарева О.Н.
}

ГБУЗ МО Московский областной научно-исследовательский клинический институт им. М.Ф. Владимирского, Москва

ЦЕЛЬ: оценить эффективность терапии деносумабом у больных с костными проявлениями первичного гиперпаратиреоза.

МАТЕРИАЛЫ И МЕТОДЫ: в наблюдение были включены больные с костными проявлениями первичного гиперпаратиреоза. Пациенты были распределены на две группы в зависимости от наличия паратиреоидэктомии в анамнезе. Всем больным исходно и каждые 6 месяцев исследовались паратгормон (ПТГ, норма 15-65 пл/мл) и общий кальций сыворотки крови (норма 2,12-2,55 ммоль/л). Также исходно и в динамике каждые 12 месяцев проводилась денситометрия (DXA) с оценкой минеральной плотности кости (МПК) позвоночника, бедра и предплечья. Для подтверждения/исключения компрессионных переломов тел позвонков всем больным выполнялась боковая рентгенография грудного и поясничного отделов позвоночника. Всем пациентам был назначен деносумаб в дозе 60мг п/к 1 раз в 6 месяцев. Продолжительность наблюдения на данный момент составила 2 года.

РЕЗУЛЬТАТЫ И ОБСУЖДЕНИЕ: В наблюденИе былИ включены 33 пациента $(2$ (6,1\%) муЖчины и 31 (93,9\%) женщина), средний возраст - 70,6 10,6 (46,0;86,0) лет. Все вошедшие в исследование женщины находились в постменопаузе продолжительностью не менее двух лет.

В группу перенесших оперативное лечение вошло 15 больных, средний возраст $-68,1 \pm 9,46$ $(50,0 ; 83,0)$ лет. Исходные лабораторные параметры соответствовали активной фазе заболевания: сред-

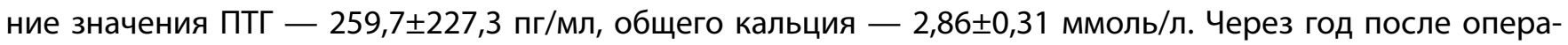
тивного лечения и на фоне приема деносумаба лабораторные показатели значимо снизились $(p<0,05)$ и находились в пределах референсных значений, что свидетельствовало о ремиссии гиперпаратиреоза: ПТГ $-57,9 \pm 27,2$ пг/мл, общий кальций $-2,46 \pm 0,18$ ммоль/л. МПК на фоне терапии деносумабом у больных с остеопорозом и ремиссией гиперпаратиреоза значимо повысились по сравнению с исходным

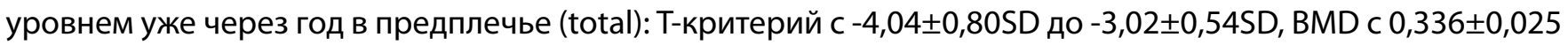
до 0,415 $\pm 0,011 г / \mathrm{cm}^{2}(\mathrm{p}=0,04)$ и через 2 года в позвоночнике: Т-критерий с $-3,35 \pm 0,92 \mathrm{SD}$ до $-2,53 \pm 0,65 \mathrm{SD}$,

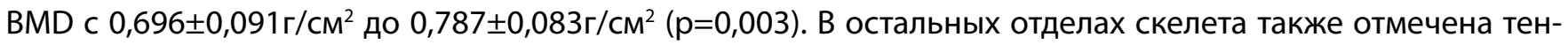
денция повышения МПК в течение всего периода наблюдения.

Лечение деносумабом без предшествующего оперативного лечения было назначено 18 больным,

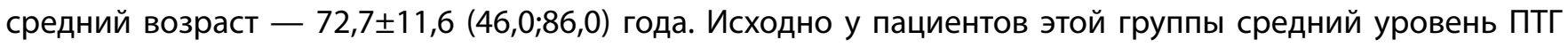

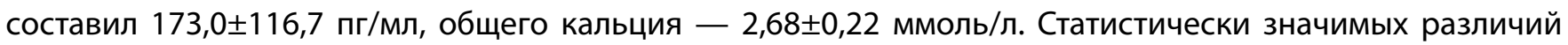
по всем параметрам между группами выявлено не было. Через год терапии деносумабом лабораторные показатели значимо не изменились по сравнению с исходными: ПТГ - 187,5 131,7 пг/мл, общий

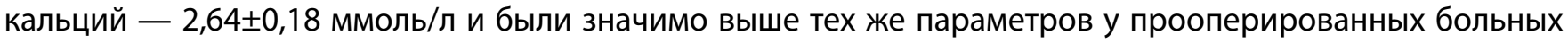
$(p<0,001)$. У пациентов с консервативной тактикой лечения также было отмечено повышение МПК всех отделов скелета в течение периода наблюдения, наиболее существенное, но статистически не значимое,

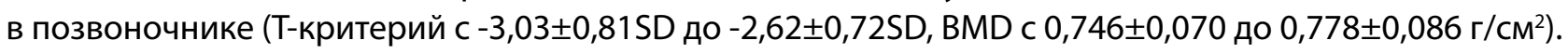

Новых переломов в период лечения у пациентов обеих групп зарегистрировано не было.

ВЫвОды: использование деносумаба у больных с костными проявлениями первичного гиперпаратиреоза способствует росту МПК при хорошем профиле переносимости лечения. 